\title{
Association of differential miRNA expression with hepatic vs. peritoneal metastatic spread in colorectal cancer
}

Sabine Heublein ${ }^{1,2}$, Markus Albertsmeier ${ }^{1}$, David Pfeifer ${ }^{1}$, Lisa Loehrs ${ }^{3}$, Alexandr V. Bazhin 1,4 ${ }^{1,}$ Thomas Kirchner ${ }^{3,4,5}$, Jens Werner ${ }^{1,4}$, Jens Neumann ${ }^{3+}$ and Martin Kurt Angele ${ }^{1 *+}$

\begin{abstract}
Background: Though peritoneal carcinomatosis reflects a late stage of colorectal cancer (CRC), only few patients present with synchronous or metachronous liver metastases alongside their peritoneal carcinomatosis. It is hypothesized that this phenomenon may be causally linked to molecular characteristics of the primary CRC. This study used miRNA profiling of primary CRC tissue either metastasized to the liver, to the peritoneum or not metastasized at all thus to identify miRNAs potentially associated with defining the site of metastatic spread in CRC.

Methods: Tissue of the primary tumor stemming from CRC patients diagnosed for either liver metastasis $(\mathrm{LM} ; n=10)$ or peritoneal carcinomatosis (PER; $\mathrm{n}=10$ ) was analyzed in this study. Advanced CRC cases without metastasis $(\mathrm{MO} ; n=3)$ were also included thus to select on those miRNAs most potentially associated with determining metastatic spread in general. miRNA profiling of 754 different miRNAs was performed in each group. MiRNAs being either differentially expressed comparing PER and LM or even triple differentially expressed (PER vs. LM vs. M0) were identified. Differentially expressed miRNAs were further validated by in silico and functional analysis.

Results: Comparative analysis identified 41 miRNAs to be differentially expressed comparing primary tumors metastasized to the liver as opposed to those spread to the peritoneum. A set of 31 miRNAs was significantly induced in primary tumors that spread to the peritoneum (PER), while the remaining 10 miRNAs were found to be repressed. Out of these 41 miRNAs a number of 25 miRNAs was triple-differentially expressed (i.e. differentially expressed comparing LM vs. PER vs. MO). The latter underwent in silico analysis. Finally, we demonstrated that miR-31 down-regulated C-MET in DLD-1 colon cancer cells.

Conclusions: This study demonstrates that CRC primary tumors spread to the peritoneum vs. metastasized to the liver display significantly different miRNA profiles. Larger patient cohorts will be needed to validate whether determination of e.g. miR-31 may aid to predict the course of disease and whether this may help to create individualized follow up or treatment protocols. To determine whether certain miRNAs may be involved in regulating the metastatic potential of CRC, functional studies will be essential.
\end{abstract}

Keywords: Colorectal cancer, miRNA, Metastasis

\footnotetext{
* Correspondence: martin.angele@med.uni-muenchen.de

${ }^{\dagger}$ Equal contributors

${ }^{1}$ Department of General, Visceral, Transplantation and Vascular Surgery, University

Hospital LMU Munich, Marachioninistrasse 15, 81377 Munich, Germany

Full list of author information is available at the end of the article
}

\section{Ciomed Central}

(c) The Author(s). 2018 Open Access This article is distributed under the terms of the Creative Commons Attribution 4.0 International License (http://creativecommons.org/licenses/by/4.0/), which permits unrestricted use, distribution, and reproduction in any medium, provided you give appropriate credit to the original author(s) and the source, provide a link to the Creative Commons license, and indicate if changes were made. The Creative Commons Public Domain Dedication waiver (http://creativecommons.org/publicdomain/zero/1.0/) applies to the data made available in this article, unless otherwise stated. 


\section{Background}

Metastatic spread is regarded a common feature of colorectal cancer (CRC). About 20\% of CRC cases present with distant metastasis even at the time of primary diagnosis [1]. Approximately $60 \%$ of patients diagnosed for advanced staged CRC is estimated to develop distant metastasis within 5 years [2]. Liver metastases account for about one third of all distant metastasis locations in CRC [3]. Another common site of distant spread is the peritoneum and about one out of four patients develops peritoneal carcinomatosis during the course of disease [4]. Importantly, although peritoneal carcinomatosis mirrors a late stage of disease, only few patients present with synchronous or metachronous hematogenous i.e. liver metastases [5, 6]. Our recent work highlighted that stem cell features of the primary tumor may direct the way of metastasis $[5,7]$. Those CRCs that will spread to the liver display a prominent cancer stem cell-like immuno-phenotype while those which will metastasize to the peritoneum do not. We hypothesized that that primary CRC cases developing solely PC but no hematogenous metastases lack the stem cell features needed for dissemination [5].

Factors related to this phenomenon by regulating mRNA stability have not been identified yet. Though miRNAs are considered to regulate metastatic potential of various cancer cells, it remains unexplored whether miRNA are involved in directing the metastatic route in CRC. In general, miRNAs represent small RNAs that majorly contribute to post-translational gene regulation. Since miRNAs may be determined by a fully automated, high throughput procedure they may become attractive for routine diagnostic in the future. Further, since miRNAs may be counteracted by sequence specific antisense oligo-nucleotides, they may evolve to be druggable targets. We used an expression platform covering a set of highly characterized miRNAs as well as several of the more recently discovered miRNAs along with the star miR* sequences. miRNA profiling of primary tumor tissue was employed to identify those miRNAs potentially being associated with determining the location of synchronic or metachronic metastatic spread in CRC.

\section{Methods}

\section{Patients}

Patients diagnosed for CRC $(n=23)$ between 1988 and 2012 were included in the study. Patients were selected from a study panel published earlier [5] and were further characterized within the current analysis. All patients underwent surgical resection of their CRC at the Department of General, Visceral, Transplantation and Vascular Surgery Surgery, University Hospital Munich (Munich, Germany). Formalin-fixed-paraffin-embedded tissue of the primary tumor was used for all the analysis described. CRC tissue underwent routine histopathological processing and examination. FFPE sample were stored under standardized conditions.

Patients were classified as either pT3 $(15 / 23)$ or pT4 $(8 / 23)$. Most patients were graded as high (14/23) at initial diagnosis. About half of all cases were male (12/23) and were diagnosed CRC that had already spread to abdominal lymph nodes (18/23). Mean age of the cohort was $65.8 \pm 12.5$ years.

Patients were retrospectively grouped into three groups according to metastasis location: group M0: CRC without metastasis formation during follow up period $(n=3)$, group LM: CRC metastasized to the liver either at initial diagnosis or during follow up without peritoneal carcinomatosis $(n=10)$, group PER: CRC spread to the peritoneum either at initial diagnosis or during follow up without liver metastasis $(n=10)$.

\section{Study design}

Patient data and tumor samples were retrieved from a patient panel that was collected prospectively and that has been published earlier by our group [5]. Samples were further characterized in the current analysis. The outcome assessed was metastasis formation after a mean follow up of $9.6 \pm 2.2$ years. During follow up 14 deaths were observed and mean overall survival was $8.4 \pm$ 2.1 years.

\section{Assay methods \\ RNA isolation, processing and multiplex $q R T-P C R$}

RNA isolation was performed on freshly sliced FFPE tissue samples. All preparation steps were performed under sterile, RNAse and DNAse free conditions. First, samples were dewaxed by xylene and washed in absolute ethanol. Representative tumor areas were extracted by microscope assisted microdissection. Dissection was supervised by an experienced senior pathologist (JN). Tumor stroma or other connective tissue was strictly excluded during sample preparation. A serial slide of each sample was $H \& E$ stained thus to ensure that areas of necrotic tumor, lymphocyte rich regions or areas directly adjacent to the invasive front do not get included. RNA was transferred into $1.5 \mathrm{ml}$ tubes and further processed by applying the RecoverAll ${ }^{\mathrm{TM}}$ Total Nucleic Acid Isolation Kit for FFPE (Applied Biosystems, Carlsbad, CA) as per manufacturer's recommendation.

Amount and quality of total RNA was quantified by a NanoDrop (Thermo Fisher Scientific, Waltham, MA) spectrophotometer. Those samples that had passed the quality control (A260/280 > 2.0, clear single RNA peak) were processed further. RNA concentrations were adjusted and equal amounts of RNA underwent cDNA synthesis using either Panel A of B multiplex primer sets (Applied Biosystems). cDNA synthesis was performed 
using the TaqMan ${ }^{\circ}$ MicroRNA Reverse Transcription Kit (Applied Biosystems) according to manufacturer's protocol. Reverse transcription was run on a Mastercycler gradient PCR machine (Eppendorf, Hamburg, Germany) using the following PCR program: $\left(2 \mathrm{~min}\right.$ at $16{ }^{\circ} \mathrm{C}$, $1 \mathrm{~min}$ at $42{ }^{\circ} \mathrm{C}, 1 \mathrm{sec}$ at $\left.50{ }^{\circ} \mathrm{C}\right) \times 40$ cycles followed by 5 min at $85{ }^{\circ} \mathrm{C}$ and cooling down to $4{ }^{\circ} \mathrm{C}$. cDNAs were pre-amplified using miRNA specific primers as provided with the Megaplex ${ }^{\mathrm{Tm}}$ Primer Pools, Human Pools Set v3.0 (Applied Biosystems). Pre-amplification was run on a Mastercycler gradient PCR machine (Eppendorf) using the following PCR program: $10 \mathrm{~min}$ at $95{ }^{\circ} \mathrm{C}, 2 \mathrm{~min}$ at $55^{\circ} \mathrm{C}, 2 \mathrm{~min}$ at $72{ }^{\circ} \mathrm{C}, 12$ amplification cycles $(15 \mathrm{~s}$ at $95^{\circ} \mathrm{C}$ and $4 \mathrm{~min}$ at $60^{\circ} \mathrm{C}$ ) followed by $10 \mathrm{~min}$ at $99.9^{\circ} \mathrm{C}$ and cooling down to $4{ }^{\circ} \mathrm{C}$.

Pre-amplified cDNA underwent single assay PCR for RNU44 and hsa-miR185-5p to check for nucleic acid integrity. Finally, equal volumes of diluted, pre-amplified cDNA, TaqMan ${ }^{\circ}$ Universal PCR Master Mix, No AmpErase ${ }^{\circ}$ UNG and DEPC-water were loaded onto TaqMan ${ }^{\circ}$ Array Human MicroRNA A + B Cards Set (Applied Biosystems). Three patient samples per group (M0, LM, PER) were pooled. qRT-PCR run was performed. MiRNAs being sufficiently expressed (i.e. cycle threshold (CT) lower than 30 - as recommended by Applied Biosystems) were selected for comparative analysis. RNU44, RNU48 and U6 snRNA were available as housekeeping genes on all TLDA cards run. Ath-miR159a served as a negative control.

The $2^{\text {-ddCT }}$ method was used to quantify relative miRNA expression $(\mathrm{dCT}$ (target sample $)=\mathrm{CT} \quad$ (target gene) - CT (housekeeping gene); ddCT (target sample) $=\mathrm{dCT}($ target sample) $-\mathrm{dCT}$ (reference sample)) [8]. A set of 25 miRNAs was found to be triple differentially expressed. Out of these 25 miRNAs three miRNAs were selected for further analysis. First, the TLDA result was validated by single PCR on three cases per group (that had been measured on the TLDA card as well) and second, expression of miR-215-5p, miR-31-5p and miR483-5p was analyzed in the whole study sample.

\section{c-MET immunohistochemistry}

c-MET immunohistochemistry (IHC) was performed on LM and PER samples. The staining method employed had been extensively validated and published by our group before [9]. In brief, c-MET (monoclonal anti-rabbit IgG, clone EP1454Y, Epitomics, Burlingame, CA) was used at a dilution of 1:150 and was detected using the Vectastain Elite ABC kit (Vector, Burlingame, CA). DAB (Dako, Glostrup, Denmark) served as a chromogen. System (without primary antibody) and isotype (unspecific rabbit IgG instead of the primary antibody) controls were performed to control for unspecific staining. c-MET IHC staining was determined using the $\mathrm{H}$-score [9].

\section{miR31 target gene validation}

A human miRNA expression vector (pEZX-MR04) encoding either hsa-miR31 or a scrambled miRNA (both from Gene Copoeia, Rockville, MD) was transfected into DLD- 1 cells by using Lipofectamine 2000 as a transfection system. Transfection was performed as per manufacturer's recommendation using a 4:1 (transfection reagent to plasmid) ratio. This ratio had been demonstrated to achieve the highest transfection efficiency (data not shown). Successful transfection of the transgene and expression of miR-31 was monitored by TaqMan qRT-PCR. RNU44 served as a house-keeping gene.

\section{Cell culture}

DLD-1 colon cancer cells were purchased from ATCC (LGC Standards GmbH, Wesel, Germany). Cell lines were routinely checked for mycoplasma contamination and cell line authentication and were found to be contamination free. RPMI1640 containing 10\% fetal calf serum was used as a standard culture media. Neither antibiotics nor antimycotics were added to culture media. Cells were passaged twice a week. Only those cultures passaged at least 4 times and maximum up to 15 times were used for the experiments.

\section{Western blotting}

Cells were seeded at a density of $75 \%$ per well and transfection was performed $24 \mathrm{~h}$ after plating. Plates were incubated 3 days at $37{ }^{\circ} \mathrm{C}$ in a humidified atmosphere and were afterwards lysed in RIPA buffer containing protease and phosphatase inhibitors. Lysates were cleared by centrifugation at $15.000 \mathrm{rpm}$ for $15 \mathrm{~min}$ at $4{ }^{\circ} \mathrm{C}$. Total protein concentrations of the supernatants were quantified and adjusted. The Mini-Protean System (Biorad, Hercules, $\mathrm{CA})$ was used for polyacrylamide gel electrophoresis and blotting. PVDF membranes were blocked in $5 \%$ milk powder in TBS-0.1\%Tween20 (TBST) for $1 \mathrm{~h}$ at room temperature. Rabbit anti-cMet (Cell Signalling Technologies; diluted $1: 1000$ in 5\% milk powder-TBST) and rabbit anti-GAPDH (Cell Signalling Technologies; diluted $1: 2000$ in 5\% milk powder-TBST) were used to incubate membranes overnight. Membranes were processed using a goat anti-rabbit IRDye ${ }^{\circ}$ 680RD secondary antibody (Cell Signalling Technologies) according to the manufacturer's instructions. Each experiment was repeated three times under the same conditions achieving similar results. Blots were quantified by employing the QuantityOne analysis software (Biorad, Hercules, CA).

\section{Statistical analysis methods}

This study has been carried out according to the REMARK (Reporting Recommendations for Tumor Marker Prognostic Studies) criteria [10]. 
The IBM statistics package SPSS (version 22) was used to test data for statistical significance. Fisher's exact test and Student's T test were used. Cell culture experiments were repeated three times achieving similar results. Replicates were performed independently and on different passages of cells. A probability value of $p<0.05$ was deemed significant in all statistical tests.

\section{Results}

\section{Study cohort}

The current analysis selected 23 cases from a larger study panel previously investigated by Neumann et al. [5] for miRNA analysis. The current study sample $(n=23)$ was characterized as follows: 10 patients diagnosed for CRC metastasized to the liver either at initial diagnosis or during follow up without peritoneal carcinomatosis (termed 'LM'), 10 patients diagnosed for CRC spread to the peritoneum either at initial diagnosis or during follow up without liver metastasis (termed 'PER') and finally three CRC patients without metastasis formation during follow up period (termed 'M0'). Patient characteristics and clinico-pathological variables according to WHO 2010 and TNM 2009 are summarized in Table 1. Gender, age, grade, UICC stage and pN-Stage were not significantly different when cases with peritoneal carcinomatosis and cases diagnosed for liver metastasis were compared. Those tumors that had spread to the peritoneum were more often assigned a pT4 grade than those which presented with liver metastasis (7/10 vs. $0 / 10 ; p=0.003)$.

A recent study from our group found that expression of a stem cell classifier ( $\beta$ Cat ${ }^{\text {high }}$ and $C D 44^{\text {high }}$ and/or $\mathrm{CD} 133^{\text {high }}$ ) in primary $\mathrm{CRC}$ tissue predicts metastatic

Table 1 Patients' characteristics

\begin{tabular}{llll}
\hline & LM & PER & M0 \\
\hline UICC & 0 & 0 & 1 \\
III & 0 & 3 & 2 \\
IV & 10 & 7 & 0 \\
Grading & & & \\
G2 & 6 & 2 & 1 \\
G3 & 4 & 8 & 2 \\
pT & & & 2 \\
pT3 & 10 & 3 & 1 \\
pT4 & 0 & 7 & \\
pN & & & 2 \\
pNo & 3 & 10 & 1 \\
pN + & 7 & 6 & 0 \\
sex & & 4 & \\
female & 4 & & \\
male & 6 & & \\
\hline
\end{tabular}

spread to the liver [5], while absence of a stem cell like immuno-phenotype was characteristic for CRC metastasized to the peritoneum. Regarding the patient cohort studied in the current work, the stem cell classifier ( $\beta C$ at ${ }^{\text {high }}$ and $C D 44^{\text {high }}$ and/or $C D 133^{\text {high }}$ ) was detected in all those primary tumors that had set liver metastasis either at time of diagnosis or during follow up (10/10). The $\beta C$ at ${ }^{\text {high }}$ and CD44 $4^{\text {high }}$ and/or CD $133^{\text {high }}$-phenotype was much rarer in cancers that presented alongside with peritoneal carcinomatosis (PER group, 2/10, $p=0.001$ ) or had not set metastasis at all (M0 group; $0 / 3, p=$ 0.003) (Fig. 1).

\section{Differential miRNA expression is associated with metastatic spread of the primary CRC}

MiRNA profiling was performed in primary colon cancer tissue of the study sample described above. Seven hundred fifty-eight miRNAs (754 target miRNAs and 4 control RNAs) were analyzed (Fig. 2a). A total number of 275 miRNAs (36.5\%) was detected with a cycle threshold (CT) lower than 30 and underwent further analysis. Out of these 275 miRNAs a number of $41 \mathrm{miR}$ NAs was identified to be differentially expressed (upregulated $>2.00$-fold or downregulated $<0.500$-fold) when cases diagnosed with liver metastasis (LM) were compared to those with peritoneal carcinomatosis (PER) (Fig. 2b). A set of 31 miRNAs was significantly upregulated in the PER group, while 10 miRNAs were found to be repressed in PER as compared to LM. The miRNAs most significantly induced in PER were hsamiR-215-5p (17-fold), hsa-miR-31-3p (8.9-fold) and hsamiR-31-5p (5.4-fold). On the opposite, miR-483-5p (0.04-fold), hsa-miR-1226-5p (0.29-fold) and hsa-miR296-5p (0.32-fold) were identified to be most obviously repressed (Fig. 2b).

As a second step, we questioned how to further select on those miRNAs potentially associated with peritoneal carcinomatosis and hence not only differentially expressed to LM but also to M0 (abbreviated as PER to M0 in Fig. 2c). A set of 27 miRNAs out of these 41 miRNAs identified previously was also found to be differentially expressed to $\mathrm{M} 0$ (Fig. 2c). hsa-miR-148a-3p (2.8-fold) and hsa-miR-215-5p (3.6-fold) were upregulated when normalized to M0, while the remaining 25 miRNAs were downregulated. The same analysis was repeated regarding differential expression of miRNAs in primary tumors metastasized to the liver (LM) as compared to tumors that did not metastasize at all (M0) (abbreviated as LM to M0 in Fig. 2d). A number of 36 miRNAs out of the 41 miRNAs described above (Fig. 2b) was also found to be differentially expressed regarding LM to M0 (Fig. 2d). Comparing LM to M0 only a single miRNA (hsa-miR-483-5p) was upregulated (7.5-fold).

Taken together comparative analysis of PER vs. LM (Fig. 2b), PER vs. M0 (Fig. 2c) and LM vs. M0 (Fig. 2d) 

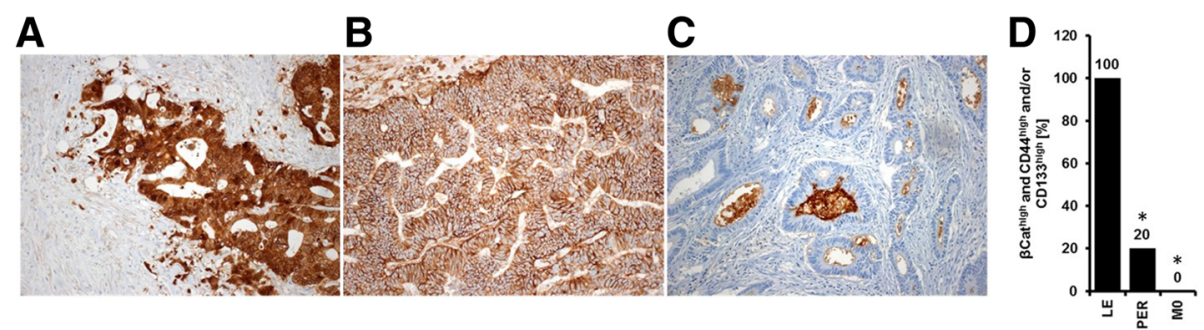

Fig. 1 beta Catenin (a), CD44 (b) and CD133 (c) immunopositivity served to sub-define primary tumors that had either spread to the peritoneum or to the liver: Presence of a stem cell like phenotype as defined by beta Catenin (a), CD44 (b) and/or CD133 (c) high immunopositivity was found to characterize primary tumors that spread to the liver, while absence of a stem cell like immuno-phenotype was characteristic for CRC metastasized to the peritoneum [5]. The current analysis selected 23 cases $(n(L M)=10, n(P E R)=10, n(M 0)=3$ ) from the study panel previously investigated by Neumann et al. for further characterization including miRNA analysis. Presence of a stem cell phenotype ( $\beta$ Cat ${ }^{\text {high }}$ and CD44 ${ }^{\text {high }}$ and/or CD133 $3^{\text {high }}$ ) in LM samples and absence of this stem cell classifier in PER and M0 samples was highly reproducible in the sub-sample analyzed in the current study (d). The stem cell phenotype was much rarer in PER $(p=0.001)$ and MO $(p=0.003)$ than in LM samples. Significant changes are indicated by stars $\left(^{*}\right)$ in $(\mathbf{d})$

revealed a set of 25 miRNAs to be triple-differentially expressed, i.e. differentially among PER vs. LM, PER vs. M0 and LM vs. M0 at the same time (Fig. 3a). These miRNAs underwent in silico prediction analysis for their association to colorectal cancer. According to the miRCancer search algorithm [11-14] out of these $25 \mathrm{miR}$ NAs being triple-differentially expressed a number of 16 miRNAs had been published to be linked to CRC in the past. Out of these hsa-miR-215-5p, hsa-miR-31-5p as well as hsa-miR-483-5p were further validated by single PCR. Results obtained from TLDA cards were found to be highly reproducible on single PCR level (Fig. 3b). hsamiR-215-5p, hsa-miR-31-5p and hsa-miR-483-5p were then analyzed on the whole patient sample. Again, hsamiR-483-5p was repressed (0.51-fold) and both hsa-miR215-5p - (3.2-fold) and hsa-miR-31-5p (12-fold) were induced (Fig. 3c). Statistical analysis comparing miRNA expression on the whole sample only proved a significant difference in case of hsa-miR-31-5p ( $p=0.002$; Fig. 3c).

\section{miR-31 reduces c-MET expression in DLD-1 colon cancer cells}

We finally questioned whether hsa-miR-31-5p may functionally interfere with metastasis formation. A literature search revealed that hsa-miR-31-5p has been reported to regulate genes involved in epithelial-to-mesenchymal transition (EMT) [15-18], which is assumed to be a major step within metastasis formation [19]. For instance, Mitamura et al. reported miR-31 to directly target c-MET - a prominent mediator of EMT - in an ovarian cancer model [15]. We thus selected c-MET to test whether miR-31 is active in colon cancer cells.

DLD-1 colon cancer cells were transfected with pEZXMR04-miR-31 or pEZX-MR04-scr control vector. Target gene expression was monitored by qRT-PCR 3, 7 and $14 \mathrm{~h}$ after transfection. Although we observed a net increase in hsa-miR-31-5p expression at all the time points analysed (3 h: 1.4-fold, 7 h: 5.8-fold, 12 h: 13-fold), statistical testing proved significant induction for the $14 \mathrm{~h}$ $(p=0.013)$ and the $7 \mathrm{~h}$ incubation period $(p=0.046)$ (Fig. 4a). As a consequence, protein expression of c-MET was measured $14 \mathrm{~h}$ after transfection and western blotting revealed significant downregulation of c-MET (0.53-fold, $p<0.001$; Fig. 4b).

Interestingly, regarding the patient panel expression of hsa-miR-31-5p was inversely correlated $(p=0.011)$ with presence of the stem cell classifier $\left(\beta C a t^{\text {high }}\right.$ and $\mathrm{CD} 44^{\text {high }}$ and/or $\mathrm{CD} 133^{\text {high }}$ ). This is consistent with the observation that the stem cell classifier has been detected in all those cancers spread to the liver which at the same time are expressing hsa-miR-31-5p on a low level (Fig. 3c). On the other hand, PER cases were negative for the stem cell classifier while abundantly expressing hsa-mir-31-5p (Figs. 1d and 3c). Although c-MET was regulated by miR-31 in DLD-1 colon cancer cells, we could not identify a significant statistical association of hsa-miR-31-5p expression and c-MET immunopositivity in the patients' tumor tissue samples.

\section{Discussion}

Determination of metastatic spread in colorectal cancer Several reports hypothesize that the risk of distant metastatic spread may be predicted from the molecular profile of the primary tumor $[5,20]$. As a future perspective, biomarkers identified in the primary tumor may aid to forecast the course of disease i.e. location of metastatic spread or whether metastasis will develop at all. Such attempt may one day aid the clinician to plan individualized follow up and potentially treatment protocols.

The probability of distant spread and poor outcome has been linked to a 17-gene signature identified across different types of human solid tumors [20]. Apart from predicting the probability of metastatic spread in general, molecular characteristics of the primary tumor may also 

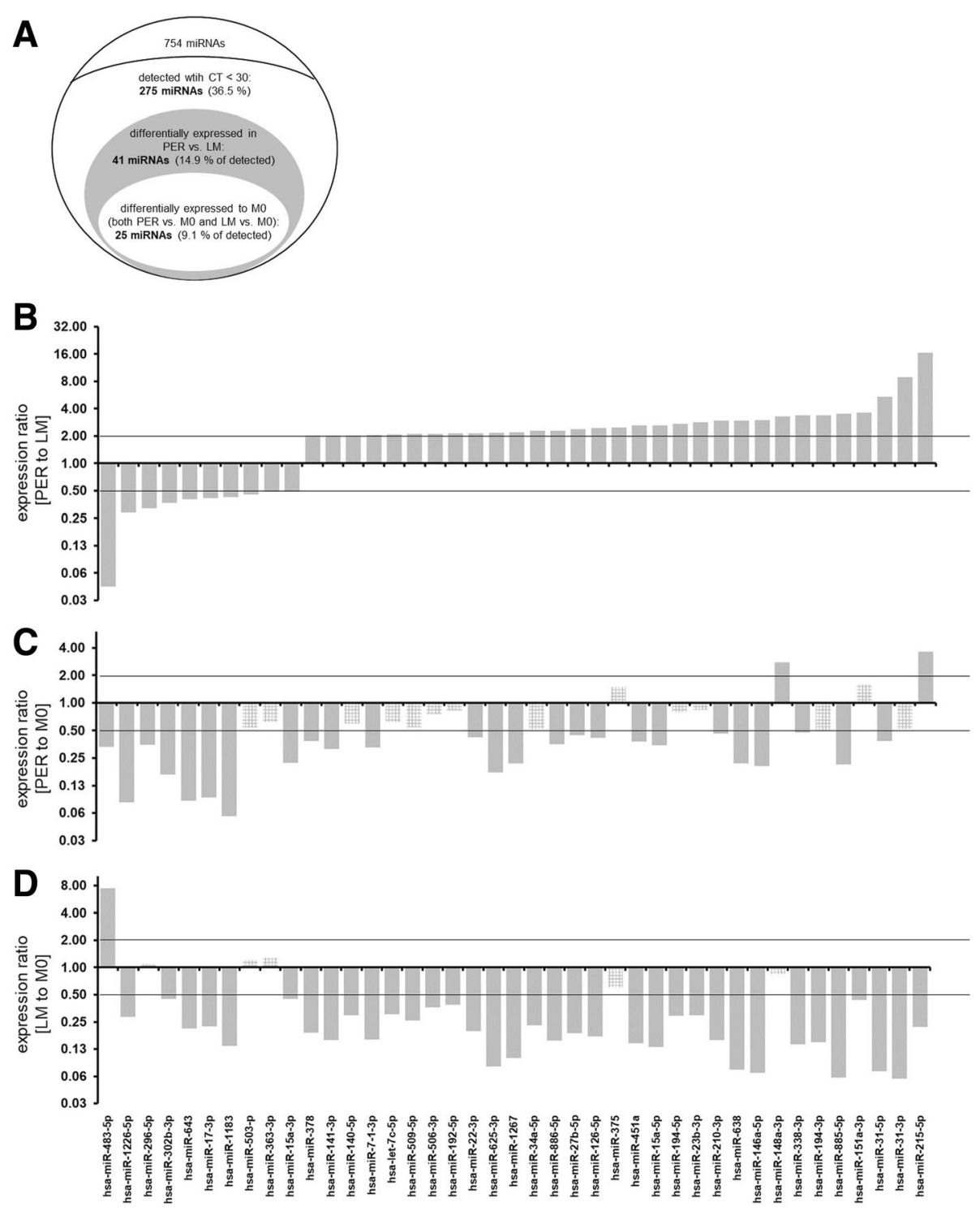

Fig. 2 miRNAs found to be differentially expressed. miRNA analysis and selection has been summarized schematically (a). miRNAs found to be differentially expressed in tumors that had spread to the peritoneum as compared with those metastasized to the liver are displayed (b). These miRNAs differentially expressed comparing LM and PER were then normalized to expression of the respective miRNA in M0 (c, d). Expression levels of PER vs. MO are shown in $\mathbf{c}$, while expression levels of LM vs. MO are shown in $\mathbf{d}$. Grey charts represent significant changes whereas squared charts stand for miRNAs not differentially expressed in the respective analysis $(\mathbf{c}, \mathbf{d})$. Expression ratios $\left(2^{\text {-ddcT }}\right)$ are displayed on a $\log 2$ scale

forecast the site of metastasis formation [5, 21]. Neumann et al. found that this phenomenon may be related to the cancer stem cell phenotype of the primary tumor and that those primary CRCs that lack stem cell like characteristics may less often set liver metastasis during their clinical course [5]. Potentially, this may be linked to the reduced potential to disseminate via the blood stream. miRNAs have been widely associated with cancer stem cell phenotype in colorectal cancer mainly via regulating Wnt/B-catenin and Notch signalling $[22,23]$. For instance, miR-215, which is among those miRNAs most often reported on in CSCs, has been demonstrated to regulate CSC differentiation and chemoresistance [23-26]. Search of miR-215 target genes revealed that miR-215 may repress expression of cell cycle and stemness genes while downregulation of miR-215 itself augments clonogenicity of colon cancer cells [24]. Interestingly, we found miR-215 (hsa-miR-215-5p) to be repressed in those primary tumors that metastasized to the liver as compared to those that spread to the peritoneum. This may support the cancer stem cell like phenotype of colorectal cancer metastasized to the liver as hypothesized in our former study [5]. 

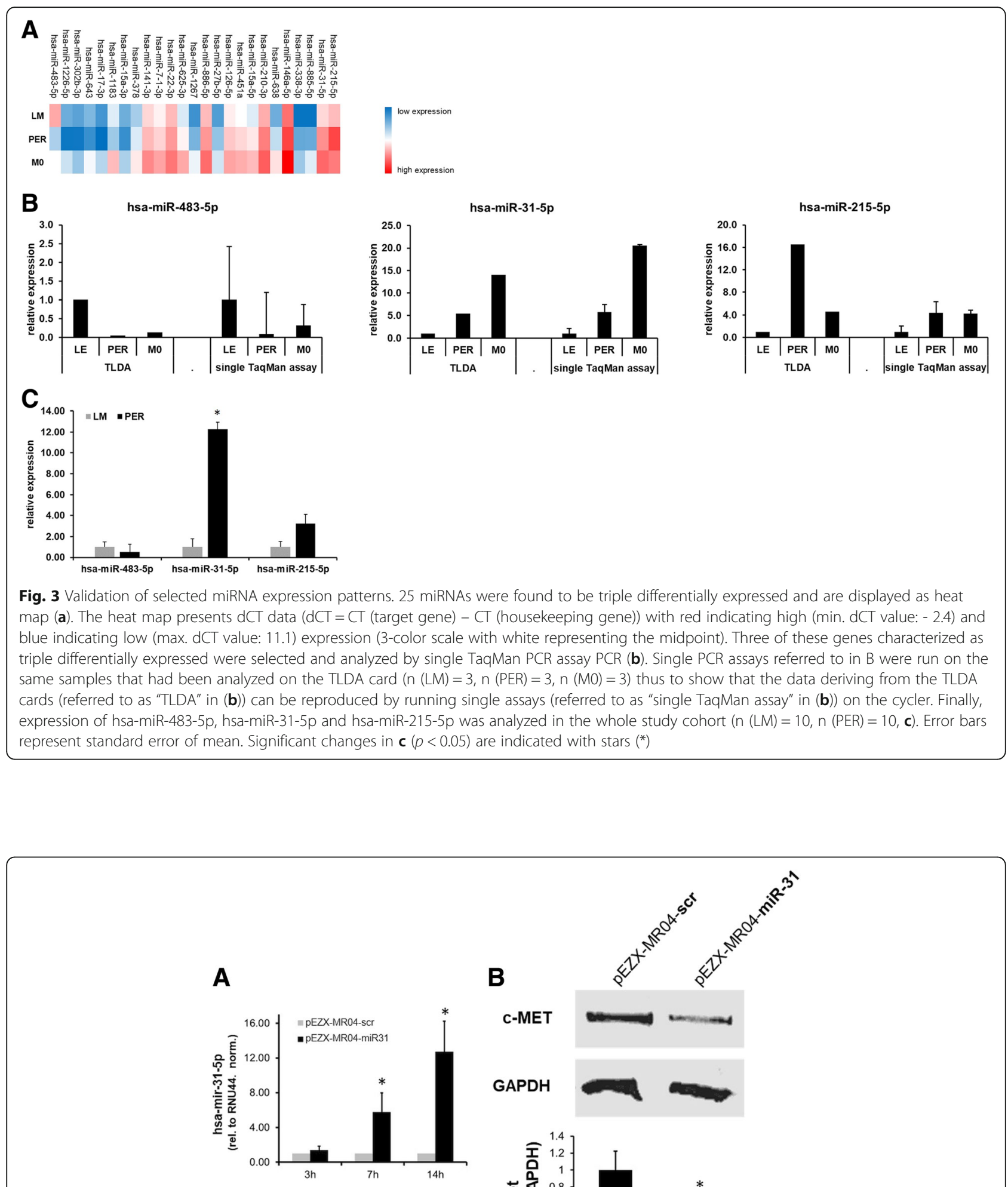

B
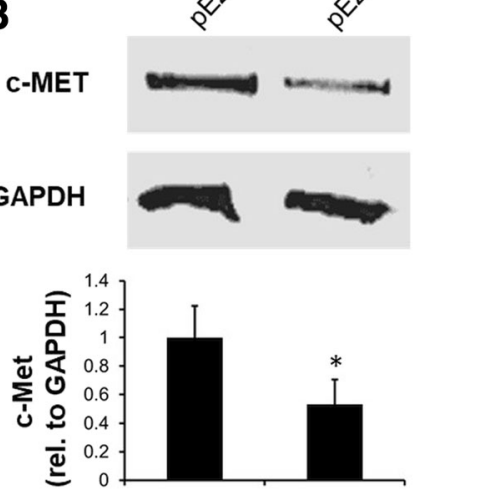

Fig. 4 Target gene regulation by miR-31. Expression of hsa-miR-31-5p in DLD-1 cells transfected with miR-31- or scr-control plasmid was monitored 3, 7 and $14 \mathrm{~h}$ after transfection by TaqMan-PCR (a). In addition, c-Met protein was determined $14 \mathrm{~h}$ after transfection by western blotting (b). Stars (*) indicate significant changes $(p<0.05)$. Data derive from three different experiments achieving similar results 
In general, research on how miRNAs may be involved in determining metastatic spread is on the increase [27]. However, previous studies compared colorectal cancer tissue to normal non-cancer controls, drawing conclusions on those miRNAs potentially involved in the biology of CRC more generally [28-30]. To the best of our knowledge, no study has been published on how miRNAs may influence the route of metastasis i.e. via the blood stream into distant organs or via local spread into the peritoneum. The current study identified a couple of miRNAs to be differentially expressed among CRC samples that set metastasis into either liver or peritoneum and stage-matched CRC cases that had not set any metastasis at all. Several miRNAs identified in our analysis had already proven to be differentially expressed in colorectal cancer or may even be associated with clinicopathological parameters [25, 27, 28]. Hence, our data may encourage functional analysis linking these miRNAs to metastasis formation in in vitro and in vivo models.

Beyond hsa-miR-215-5p another two miRNAs were validated on the whole patient panel. We selected hsamiR-483-5p, since it was strongest repressed miRNA when expression of PER vs. LM was compared (Fig. $2 b$ ). In contrast, hsa-miR-31-5p was chosen since it was the second most obviously upregulated (regarding PER to LM, Fig. 2b) miRNA. hsa-miR-31-5p underwent functional analysis due to three reasons. When the analysis was extended on the whole patient sample, differential expression only remained significant in case of hsa-miR-31-5p (Fig. 3c). In addition, its star strand, i.e. hsa-miR-31-3p, was also differentially expressed, though failed to fulfil the criteria of triple differential expression. Interestingly, up-regulation of both hsamiR-31-5p and its passenger strand hsa-miR-31-3p was detected to be predictive in metastatic CRC patients treated with cetuximab - again suggesting this miRNA to play a dominant role in metastatic CRC [31]. Finally, miR-31 has been demonstrated to regulate cMet in ovarian cancer [15].

Of course, demonstrating inverse correlation of miR-31 and c-MET at the exact time point (!) of metastatic spread would be desirable. However, about half of our primary tumor samples were resected years before metastatic spread was diagnosed (i.e. metachronous metastatic spread) and thus our samples did not mirror the state or time point of metastatic spread. Since both metastasis formation and miRNA mediated gene regulation are highly dynamic processes, determination of c-MET at surgery of the primary tumor, i.e. years before metastasis formation may take place, seems not to be useful in terms of analyzing whether miR-31 may regulate c-MET. This may explain why there was no significant statistical association of miR-31 and c-MET immunopositivity in our tumor samples. An additional reason for this is that initiation of metastasis formation is a complex process and is supposed to be restricted to stem cells or small sub-populations within the tumor [32-34]. Hypothetically assuming that miR-31 regulates c-MET in cancer cells thus to participate in determining metastatic spread, such a regulation process may only take place in single clones that initiate metastasis formation. The method used to determine c-MET (= single marker $\mathrm{IHC})$ in the current study was not suited to discriminate metastasis initiating sub-clones from the rest of the tumor cells. Hence, we quantified c-MET throughout the whole tumor sample rather than in specific sub-populations. This may methodically mask potential regulatory effects of miR-31 on c-MET in certain sub-populations of tumor cells.

\section{Hsa-miR-31-5p may act on epithelial-to-mesenchymal transition by target gene regulation}

Epithelial-to-mesenchymal transition (EMT) is regarded to be a major step within metastasis formation [19]. miR-31 has been demonstrated to regulate a number of genes closely associated with EMT and metastasis formation. For instance, miR-31 was found to inhibit SATB2 thereby supporting CRC cell growth, invasion and metastasis formation [16]. On the other hand, miR-31 overexpression has been shown to inhibit autophagy in cancer associated fibroblasts of CRC thereby increasing apoptosis and reducing migration of co-cultured CRC cell lines [17]. Besides this observation, autophagy rate may also serve as a readout parameter for miR-31 activity - even in FFPE samples. Induction of miR-31 inhibited integrin alpha $\mathrm{V}$ and thus reduced metastatic potential of gastric cancer cells [18]. In line with this, several authors already highlighted miR-31 to directly target c-MET (also known as scatter factor) - a prominent mediator of EMT [15, 35, 36]. The current work was able to reproduce this finding in colon cancer cells. Hence, it may be hypothesized that repression of hsa-miR-31$5 p$ in LE samples may lead to up-regulation of c-MET - at least at the time of initial metastatic spread. Whether repression of c-MET by hsa-miR-31$5 \mathrm{p}$ may also support EMT and whether this may aid tumors cells to spread via the blood stream needs to be determined. Finally, the current study demonstrated induction of hsa-miR-31-5p in those primary tumors that spread to the peritoneum - again supporting the hypothesis that hsa-miR-31may regulate metastatic potential of CRC.

\section{Conclusion}

This study found that miRNA expression of colorectal cancer primary tumor tissue may predict the site of 
synchronous or metachronous metastatic spread. We further identified hsa-miR-31-5p to be overexpressed in those CRC cases that were diagnosed with peritoneal carcinomatosis either at initial diagnosis or during follow up. Interestingly, miR31 has been shown to repress expression of c-MET [15] - a kinase known to play a pivotal role in epithelial-to-mesenchymal-transition and hematogenous metastatic spread [37, 38].

\section{Abbreviations}

CRC: Colorectal cancer; EMT: Epithelial-to-mesenchymal transition; LM: Liver metastasis; MO: No distant metastasis; miRNA: micro RNA; PER: Peritoneal carcinomatosis; PVDF: Polyvinylidene fluoride; TLDA: TaqMan Low Density Array

\section{Acknowledgements}

The authors would like to thank Mrs. Anne Wagner for excellent technical support.

The full miRNA name as recommended by miRBase ID (v20) was used whenever possible. However, not all the papers cited provided full miRNA names - most potentially referring to the leading strand without mentioning that in particular. Therefore - especially within the discussion - abbreviated miRNA (i.e. miR-215 instead of has-miR-215-5p) names were used for increasing readability of the text.

\section{Funding}

This project was funded by a grant of the Weigand-Stiftung to MKA and JN. The funder had no role in study design, data collection and analysis, decision to publish or preparation of the manuscript.

\section{Availability of data and materials}

The datasets used and/or analyzed during the current study available from the corresponding author on reasonable request.

\section{Authors' contribution}

SH: Participated in the design and coordination of the study, performed miRNA-expression and cell culture assays, performed the statistical analysis and drafted the manuscript. MA: Participated in the design and coordination of the study and carefully reviewed the manuscript for important intellectual content. DP: Significantly contributed to experimental analysis. LL: Participated in the selection of the cases. AB: Participated in the design and coordination of the study and carefully reviewed the manuscript for important intellectual content. TK: Participated in the design, provided funding and helped to draft the manuscript. JW: Participated in the design, provided funding and helped to draft the manuscript. JN: Is one of the principal investigators, participated in the design and coordination of the study, provided funding, participated in the selection and validation of cases and reviewed the manuscript. MKA: Is one of the principal investigators, conceived of the study and participated in its design and coordination, provided funding and approved the final version of the manuscript. All authors read and approved the final manuscript.

\section{Ethics approval and consent to participate}

This study was carried out according to the recommendations of the ethics committee of the Medical Faculty of the Ludwig-Maximilians-University Munich, Germany. The current study has been performed in a retrospective manner in a cohort of patients diagnosed and treated according to national guidelines. In addition, the data set as well as the specimens were irreversibly anonymized prior to inclusion in the study. Hence, under the circumstances aforementioned, neither a written consent nor a project specific approval by the ethic committee was necessary.

The study was performed according to the standards set in the declaration of Helsinki 1975. All researchers were blinded from patient data during experimental analysis.

\section{Consent for publication}

This study used tumor tissue that had initially been collected for histopathological diagnostics. At the time, the tissue was examined for the current study all diagnostic procedures had already been fully completed and the tissue used was thus classified as left-over material. All patient data were fully anonymized. As per declaration of our ethics committee (Ludwig-Maximilians-University Medical School (Munich, Germany)) no written informed consent of the participants is needed given the circumstances described above.

\section{Competing interests}

The authors declare that they have no competing interests.

\section{Publisher's Note}

Springer Nature remains neutral with regard to jurisdictional claims in published maps and institutional affiliations.

\section{Author details}

'Department of General, Visceral, Transplantation and Vascular Surgery, University Hospital LMU Munich, Marachioninistrasse 15, 81377 Munich, Germany. ${ }^{2}$ Department of Obstetrics and Gynaecology, Heidelberg University Hospital, Heidelberg, Germany. Institute of Pathology, Ludwig-Maximilians-Universität München, Munich, Germany. ${ }^{4}$ German Cancer Consortium (DKTK), Heidelberg, Germany. ${ }^{5}$ German Cancer Research Center (DKFZ), Heidelberg, Germany.

Received: 13 January 2017 Accepted: 24 January 2018

Published online: 20 February 2018

\section{References}

1. Cook AD, Single R, McCahill LE. Surgical resection of primary tumors in patients who present with stage IV colorectal cancer: an analysis of surveillance, epidemiology, and end results data, 1988 to 2000. Ann Surg Oncol. 2005;12(8):637-45.

2. Desch $C E$, Benson $A B$ 3rd, Somerfield MR, Flynn PJ, Krause C, Loprinzi $C L$, Minsky BD, Pfister DG, Virgo KS, Petrelli NJ, et al. colorectal cancer surveillance: 2005 update of an American Society of Clinical Oncology practice guideline. Journal of clinical oncology : official journal of the American Society of Clinical Oncology. 2005;23(33):8512-9.

3. Manfredi S, Lepage C, Hatem C, Coatmeur O, Faivre J, Bouvier AM. Epidemiology and management of liver metastases from colorectal cancer. Ann Surg. 2006:244(2):254-9.

4. Segelman J, Granath F, Holm T, Machado M, Mahteme H, Martling A. Incidence, prevalence and risk factors for peritoneal carcinomatosis from colorectal cancer. Br J Surg. 2012;99(5):699-705.

5. Neumann J, Lohrs L, Albertsmeier M, Reu S, Guba M, Werner J, Kirchner T, Angele M. Cancer stem cell markers are associated with distant Hematogenous liver metastases but not with peritoneal Carcinomatosis in colorectal cancer. Cancer Investig. 2015;33(8):354-60.

6. Thomassen I, van Gestel YR, Lemmens VE, de Hingh IH. Incidence, prognosis, and treatment options for patients with synchronous peritoneal carcinomatosis and liver metastases from colorectal origin. Dis Colon rectum. 2013:56(12):1373-80

7. Neumann J, Horst D, Kriegl L, Maatz S, Engel J, Jung A, Kirchner T. A simple immunohistochemical algorithm predicts the risk of distant metastases in right-sided colon cancer. Histopathology. 2012;60(3):416-26.

8. Livak KJ, Schmittgen TD. Analysis of relative gene expression data using real-time quantitative PCR and the 2(-Delta Delta C(T)) method. Methods. 2001;25(4):402-8.

9. Siemens H, Neumann J, Jackstadt R, Mansmann U, Horst D, Kirchner T, Hermeking $\mathrm{H}$. Detection of miR-34a promoter methylation in combination with elevated expression of c-MET and beta-catenin predicts distant metastasis of colon cancer. Clin Cancer Res. 2013;19(3):710-20.

10. LM MS, Altman DG, Sauerbrei W, Taube SE, Gion M, Clark GM. reporting recommendations for tumor marker prognostic studies. Journal of clinical oncology : official journal of the American Society of Clinical Oncology. 2005;23(36):9067-72

11. Xie B, Hochberg R, Ding Q, Wu D: miRSAT \& miRCDB: an integrated MicroRNA Sequesce analysis tool and a cancer-associated MicroRNA database; 2010.

12. Xie B, Ding Q, Han H, Wu D. miRCancer: a microRNA-cancer association database constructed by text mining on literature. Bioinformatics. 2013; 29(5):638-44.

13. Boya $X$, Qin D, Di W. Text mining on big and complex biomedical literature. In: Baoying W, Ruowang L, William P, editors. Big Data Analytics in Bioinformatics and Healthcare. Hershey: IGI Global; 2015. p. 21-45. 
14. http://mircancer.ecu.edu/search.jsp last Access 12 Aug 2017.

15. Mitamura T, Watari H, Wang L, Kanno H, Hassan MK, Miyazaki M, Katoh Y, Kimura T, Tanino M, Nishihara $H$, et al. Downregulation of miRNA-31 induces taxane resistance in ovarian cancer cells through increase of receptor tyrosine kinase MET. Oncogene. 2013;2:e40.

16. Yang $M H, Y u$ J, Chen N, Wang XY, Liu XY, Wang S, Ding YQ. Elevated microRNA-31 expression regulates colorectal cancer progression by repressing its target gene SATB2. PLoS One. 2013;8(12):e85353.

17. Yang X, Xu X, Zhu J, Zhang S, Wu Y, Wu Y, Zhao K, Xing C, Cao J, Zhu H, et al. miR-31 affects colorectal cancer cells by inhibiting autophagy in cancerassociated fibroblasts. Oncotarget. 2016;7(48):79617-28.

18. Zhang XB, Song L, Wen HJ, Bai XX, Li ZJ, Ma LJ. Upregulation of microRNA31 targeting integrin alpha5 suppresses tumor cell invasion and metastasis by indirectly regulating PI3K AKT pathway in human gastric cancer SGC7901 cells. Tumour biology : the journal of the International Society for Oncodevelopmental Biology and Medicine. 2016;37(6):8317-25.

19. Smith BN, Bhowmick NA. Role of EMT in metastasis and therapy resistance. Journal of clinical medicine. 2016;5(2):17.

20. Ramaswamy S, Ross KN, Lander ES, Golub TR. A molecular signature of metastasis in primary solid tumors. Nat Genet. 2003;33(1):49-54.

21. Minn AJ, Kang Y, Serganova I, Gupta GP, Giri DD, Doubrovin M, Ponomarev V, Gerald WL, Blasberg R, Massague J. Distinct organ-specific metastatic potential of individual breast cancer cells and primary tumors. J Clin Invest. 2005;115(1):44-55.

22. Yu Y, Nangia-Makker $P$, Farhana L, GR S, Levi E, Majumdar AP. miR-21 and miR-145 cooperation in regulation of colon cancer stem cells. Mol Cancer. 2015;14:98.

23. Mamoori A, Gopalan V, Smith RA, Lam AK. Modulatory roles of microRNAs in the regulation of different signalling pathways in large bowel cancer stem cells. Biology of the cell / under the auspices of the European Cell Biology Organization. 2016;108(3):51-64.

24. Jones MF, Hara T, Francis P, Li XL, Bilke S, Zhu Y, Pineda M, Subramanian M, Bodmer WF, Lal A. the CDX1-microRNA-215 axis regulates colorectal cancer stem cell differentiation. Proc Natl Acad Sci U S A. 2015;112(13):E1550-8.

25. Wang J, Du Y, Liu X, Cho WC, Yang Y. MicroRNAs as regulator of signaling networks in metastatic colon cancer. Biomed Res Int. 2015;2015:823620.

26. Song B, Wang Y, Titmus MA, Botchkina G, Formentini A, Kornmann M, Ju J. Molecular mechanism of chemoresistance by miR-215 in osteosarcoma and colon cancer cells. Mol Cancer. 2010;9:96.

27. Perilli L, Pizzini S, Bisognin A, Mandruzzato S, Biasiolo M, Facciolli A, Rossi E, Esposito G, Rugge M, Pilati P, et al. Human miRNome profiling in colorectal cancer and liver metastasis development. Genomics data. 2014;2:184-8.

28. Wang YX, Zhang XY, Zhang BF, Yang CQ, Chen XM, Gao HJ. Initial study of microRNA expression profiles of colonic cancer without lymph node metastasis. J Dig Dis. 2010;11(1):50-4.

29. Luo X, Burwinkel B, Tao S, Brenner H. MicroRNA signatures: novel biomarker for colorectal cancer? Cancer epidemiology, biomarkers \& prevention : a publication of the American Association for Cancer Research, cosponsored by the American Society of Preventive Oncology. 2011;20(7):1272-86.

30. Schetter AJ, Leung SY, Sohn JJ, Zanetti KA, Bowman ED, Yanaihara N, Yuen ST, Chan TL, Kwong DL, Au GK, et al. MicroRNA expression profiles associated with prognosis and therapeutic outcome in colon adenocarcinoma. JAMA. 2008;299(4):425-36.

31. Mlcochova J, Faltejskova-Vychytilova P, Ferracin M, Zagatti B, Radova L, Svoboda M, Nemecek R, John S, Kiss I, Vyzula R, et al. MicroRNA expression profiling identifies miR-31-5p/3p as associated with time to progression in wild-type RAS metastatic colorectal cancer treated with cetuximab. Oncotarget. 2015;6(36):38695-704.

32. Gerashchenko TS, Zavyalova MV, Denisov EV, Krakhmal NV, Pautova DN, Litviakov NV, Vtorushin SV, Cherdyntseva NV, Perelmuter VM. Intratumoral morphological heterogeneity of breast cancer as an indicator of the metastatic potential and tumor Chemosensitivity. Acta Nat. 2017;9(1):56-67.

33. Ong BA, Vega KJ, Houchen CW. Intestinal stem cells and the colorectal cancer microenvironment. World J Gastroenterol. 2014;20(8):1898-909.

34. Basu S, Haase G, Ben-Ze'ev A. Wnt signaling in cancer stem cells and colon cancer metastasis. F1000Res. 2016;5. Epub 2016/05/03. https://doi.org/10. 12688/f1000research.7579.1. PubMed PMID: 27134739; PubMed Central PMCID: PMCPMC4841194.

35. Bayrak OF, Gulluoglu S, Aydemir E, Ture U, Acar H, Atalay B, Demir Z, Sevli S, Creighton $C J$, Ittmann $M$, et al. MicroRNA expression profiling reveals the potential function of microRNA-31 in chordomas. J Neuro-Oncol. 2013; 115(2):143-51.

36. Hou C, Sun B, Jiang Y, Zheng J, Yang N, Ji C, Liang Z, Shi J, Zhang R, Liu Y, et al. MicroRNA-31 inhibits lung adenocarcinoma stem-like cells via downregulation of MET-PI3K-Akt signaling pathway. Anti Cancer Agents Med Chem. 2016;16(4):501-18.

37. Thiery JP, Acloque H, Huang RY, Nieto MA. Epithelial-mesenchymal transitions in development and disease. Cell. 2009;139(5):871-90.

38. Cao H, Xu E, Liu H, Wan L, Lai M. Epithelial-mesenchymal transition in colorectal cancer metastasis: a system review. Pathol Res Pract. 2015; 211(8):557-69.

\section{Submit your next manuscript to BioMed Central and we will help you at every step:}

- We accept pre-submission inquiries

- Our selector tool helps you to find the most relevant journal

- We provide round the clock customer support

- Convenient online submission

- Thorough peer review

- Inclusion in PubMed and all major indexing services

- Maximum visibility for your research

Submit your manuscript at www.biomedcentral.com/submit
Biomed Central 